\title{
Solution of Nuclear Electrodynamics Equations Taking into Account $\beta$-Charged Eradiation
}

\author{
V. Yu. Tertychny-Dauri \\ Department of Physics and Engineering, Saint-Petersburg National Research University of Information Technologies, Mechanics \\ and Optics, Saint-Petersburg, Russia \\ Email: tertychny-dauri@mail.ru
}

How to cite this paper: Tertychny-Dauri, V.Yu. (2017) Solution of Nuclear Electrodynamics Equations Taking into Account $\beta$-Charged Eradiation. Open Access Library Journal, 4: e3794.

https://doi.org/10.4236/oalib.1103794

Received: July 1, 2017

Accepted: July 23, 2017

Published: July 27, 2017

Copyright (c) 2017 by author and Open Access Library Inc.

This work is licensed under the Creative Commons Attribution International License (CC BY 4.0).

http://creativecommons.org/licenses/by/4.0/

\begin{abstract}
The study of own fields and charged particles motion and also charged fission splinters of a heavy nucleuses into nonrelativistic approximation is the subject of this article research. The main efforts are concentrated in quest of charged share components by the radioactive $\beta$-disintegration. The corresponding field equations and equations of motion in the nuclear electrodynamics processes are obtained and their solutions are found. Analysis of the microscopic equations is generalized to the level of the macroscopic description of continuous medium electrodynamics and is accompanied by quantum mechanical additions. The obtained theoretical results can be a basis for the further mathematical modelling of generation phenomenon of powerful directional electromagnetic fields on the toroid with external winding in the process of chain reaction fission and for the further creation of the nuclear electrogenerator (nuclegen) as a peculiar analogy to the quantum generator.
\end{abstract}

\section{Subject Areas}

Theoretical Physics, Modern Physics, Nuclear Physics

\section{Keywords}

Equations of Nuclear Electrodynamics, Charged Particles, Charged Fission Splinters

\section{Introduction}

At the beginning we should stop briefly on the structural description and the content of article sections. Sections 2 - 5 are devoted to the coverage of a behaviour for dotted charged particles and fission splinters within the classical nonrelativistic nuclear electrodynamics.

In Sections 2 and 3 the consecutive microscopic description on the level of 
Maxwell-Lorenz fields equations and Newton-Lorenz equations of motion is carried out. Obtained in Section 2 results serve a foundation to the conclusion of laws for nonrelativistic nuclear electrodynamics of the charged fission splinters (Sections 3 and 4) and laws for continuous medium electrodynamics with the aid of macroscopic approach in consideration of statistical description (Section 5).

In Section 6 the nonrelativistic electrodynamic model is supplemented by consideration more realistic scheme connected with quantumomechanical conclusion of the microscopic equations for fields and motions of charged particles and charged fission splinters.

The important peculiarity of presented work is a definition and solution of fields equations and motion equations for fission splinters in consideration of charged share of radioactive inside nuclon $\beta$-disintegration effect [1], i.e. in consideration of emited cascaded electrons but without consideration of $\gamma$-eradiation [2] [3] [4]. We propose the toroid is the solenoid with the external winding. Moreover inside of this toroid all fragments of the nuclear disintegration remain in force. That is why light charges don't may escape the system and therefore enter the formalism. Theoretical results of Sections $2-6$ can be also the basis for the elaboration of possible mechanism of hypermotion realization [5]-[12].

Similar to quantum generation phenomenon and some other physical effects going on intensive avalance-like scheme there are analogous processes occur by the chain fission of a heavy nucleuses [5] [9] [13] [14]: the vigorous growth of total number of neutrons, charged particles and charged fission splinters having enormous kinetic energy of motion is observed. This known phenomenon (charges motion in vacuum with great speed) is put into basis of nuclear electrodynamic effect.

On this theory principles may be constructed superpowerful energetical devices operating no on thermal interaction of various substances but exclusively on a basis of physical electronuclear conversions. Actually, it is matter of on principle new stage into energy development which at this point is named the nuclear electrodynamics.

As at laser technology the nuclear electrodynamic model for own functional beginning demands the preliminary " power reprimand" in a state of external directional electromagnetic field for making of anisotropic backcloth with the passing of chain nuclear fission reaction in vacuum.

We suppose that the nuclear electrogenerator of direct action can be highly effective and reliable alternative to contemporary thermal nuclear reactor in the future. What is more, its role and meaning in subsequent wide and speedy pioneering of outer space, to all appearance, will be predominating as a basis component of arised compact hyperreactive accelerators [5] [9].

\section{Fields and Motion of Charged Fission Particles}

We are interested the calculation recipe of electric $E_{*}(R, t) \in \mathbf{R}^{3}$ and magnetic $B_{*}(R, t) \in \mathbf{R}^{3}$ fields in the point with coordinate $R \in \mathbf{R}^{3}$ at the time moment $t$ 
which are produced by the point-particles with charges $Z_{i}$, three-dimensional coordinates $R_{i}(t)$ and velocities $\dot{R}_{i}(t)=v_{i}(t), i=1,2, \cdots$. It is known that given vector fields into Gaussian units system (taking into account of values in order $1 / c$, where $c$ is velocity of light, and of multiplicator $4 \pi$ in the expression for potentials) can be written with the aid of microcsopic MaxwellLorenz equations system in the following form

$$
\begin{gathered}
\nabla E_{*}=\sum_{i} Z_{i} \delta\left(R_{i}-R\right), \\
-E_{*}^{\prime}+\nabla \times B_{*}=\frac{1}{c} \sum_{i} Z_{i} \dot{R}_{i} \delta\left(R_{i}-R\right), \\
\nabla B_{*}=0, \quad B_{*}^{\prime}+\nabla \times E_{*}=0 .
\end{gathered}
$$

Here we denote: for the vector $\nabla$ (the differential Hamiltonian operator) is the differentiation on three-dimensional spatial coordinate $R$; the feature on top is the differentiation on $c$, notes $x y$ and $x \times y$ correspondingly mean scalar and vector products; $\delta\left(R_{i}-R\right)$ is the delta-function from $R_{i}-R$ including in the field sources.

To find solutions of Equation (1) introduce into consideration potentials. From the third Equation (1) we have for the vector potential $a(R, t)$ :

$$
B_{*}=\nabla \times a .
$$

Taking into account the fourth Equation (1) we obtain for the scalar potential $\varphi(R, t)$ :

$$
E_{*}=-\nabla \varphi-a^{\prime}
$$

After substitution of relations (2) and (3) into the first two Equation (1) ignoring of values in order $1 / c^{2}$ we derive

$$
\begin{gathered}
\nabla \varphi+\nabla^{\prime} a=-\sum_{i} Z_{i} \delta\left(R_{i}-R\right), \\
\nabla a-\nabla\left(\nabla a+\varphi^{\prime}\right)=-\frac{1}{c} \sum_{i} Z_{i} \dot{R}_{i} \delta\left(R_{i}-R\right),
\end{gathered}
$$

where $\Delta=\nabla \nabla=\nabla^{2}$ is the Laplace operator.

Relations (2), (3) determine potentials $a$ and $\varphi$ ambiguously to an approximation of the calibrated equalities

$$
a_{s}=a+\nabla \psi, \quad \varphi_{s}=\varphi-\psi^{\prime},
$$

where $\psi$ is the some arbitrary function. Take advantage of given fact so that potential $a$ and $\varphi$ satisfied the Lorenz calibrated condition

$$
\nabla a+\varphi^{\prime}=0
$$

which permits Equations (4) to write in form of two independent Poisson equations on $\varphi$ and $a$. Ignoring of values in order $1 / c^{2}$ then we obtain

$$
\Delta \varphi=-\sum_{i} Z_{i} \delta\left(R_{i}-R\right), \quad \Delta a=-\frac{1}{c} \sum_{i} Z_{i} \dot{R}_{i} \delta\left(R_{i}-R\right) .
$$

If for solution of this equations according to the work [2] to make use of relation 


$$
\Delta \cdot \frac{1}{|r|}=-4 \pi \delta(r)
$$

where $r=r(x, y, z)$ is vector-radius of the point with coordinates $x, y, z$ and $|r|=\left(x^{2}+y^{2}+z^{2}\right)^{1 / 2}$ is its the Euclidean length, then we may write potentials

$$
\varphi=\sum_{i} \frac{Z_{i}}{4 \pi \sigma_{i}}, \quad a=\frac{1}{c} \sum_{i} \frac{Z_{i} R_{i}}{4 \pi \sigma_{i}} .
$$

Further, we shall use relations (2) and (3). Then the nonrelativistic electromagnetic fields can be found as

$$
\begin{gathered}
E_{*}=\sum_{i} E_{i}, \quad E_{i}=-\frac{\nabla Z_{i}}{4 \pi \sigma_{i}}, \\
B_{*}=\sum_{i} B_{i}, \quad B_{i}=\frac{1}{c} \nabla \times \frac{Z_{i} \dot{R}_{i}}{4 \pi \sigma_{i}},
\end{gathered}
$$

where through $\sigma_{i}=\left|R_{i}-R\right|$ is denoted the distance between points with vector coordinates $R_{i}$ and $R$ in space $\mathbf{R}^{3}$.

Pay attention to that from relations (5) it follows that the field $E_{*}$ is noncirculatory. Then in fourth equation of system (1) the value $B_{*}^{\prime}$ has a order $1 / c^{2}$ and by it may ignore in considered nonrelativistic variant. Therefore in system (1) last equation may write as $\nabla \times E_{*}=0$.

Denote $\left(E\left(R_{i}, t\right), B\left(R_{i}, t\right)\right)$ the full electromagnetic field in the point $R_{i}$ at time moment $t$. This field is the sum of external field $\left(E_{0}\left(R_{i}, t\right), B_{0}\left(R_{i}, t\right)\right)$ and internal fields of the charged particles $\left(E_{*}\left(R_{i}, t\right), B_{*}\left(R_{i}, t\right)\right)$ in the given point.

The equation of $i$-th particle motion with mass $m_{i}$ under the Lorenz force influence has a form

$$
m_{i} \ddot{R}_{i}=Z_{i}\left[E\left(R_{i}, t\right)+\frac{1}{c} \dot{R}_{i} \times B\left(R_{i}, t\right)\right],
$$

where the notation

$$
\begin{gathered}
E\left(R_{i}, t\right)=\sum_{j} E_{j}\left(R_{i}, t\right)+E_{0}\left(R_{i}, t\right), \\
B\left(R_{i}, t\right)=\sum_{j} B_{j}\left(R_{i}, t\right)+B_{0}\left(R_{i}, t\right)
\end{gathered}
$$

is used, moreover $i \neq j, \sum_{j} E_{j}=E_{*}, \sum_{j} B_{j}=B_{*}$. The equation of motion (6) for nonrelativistic case taking into account the second line of the system (5) can be made more precise, namely

$$
m_{i} \ddot{R}_{i}=Z_{i}\left[E\left(R_{i}, t\right)+\frac{1}{c} \dot{R}_{i} \times B_{0}\left(R_{i}, t\right)\right],
$$

where

$$
E\left(R_{i}, t\right)=-\sum_{j} \nabla_{i} \frac{Z_{j}}{4 \pi \sigma_{i j}}+E_{0}\left(R_{i}, t\right), \quad B\left(R_{i}, t\right)=B_{0}\left(R_{i}, t\right),
$$

$\sigma_{i j}=\left|R_{i}-R_{j}\right|, i \neq j, \quad \nabla_{i}$ is partial derivation on elements of the vector $R_{i}$.

Thus, Equation (7) describes nonrelativistic motion of $i$-th charged particle, 
$i=1,2, \cdots$, in electromagnetic field of external sources and from the direction of other $j$-th particles, $j=1,2, \cdots, j \neq i$, in the given point $R_{i}$. Equation (7) can be attached to the canonical form

$$
\frac{\partial H}{\partial P_{i}}=\dot{R}_{i}, \quad \frac{\partial H}{\partial R_{i}}=-\dot{P}_{i}
$$

with aid of the Hamiltonian

$$
H=\sum_{i} \frac{P_{i}^{2}}{2 m_{i}}+\sum_{i} \sum_{j} \frac{Z_{i} Z_{j}}{8 \pi \sigma_{i j}}+\sum_{i} Z_{i}\left[\varphi_{0}\left(R_{i}, t\right)-\frac{P_{i}}{c m_{i}} \cdot A_{0}\left(R_{i}, t\right)\right], \quad i \neq j,
$$

into the canonical terms of impulse variables $P_{i}$, coordinate variables $R_{i}$ and potentials (scalar $\varphi_{0}$ and vector $A_{0}$ ) of external field.

\section{Fields and Motion of the Charged Fission Splinters}

Let us set the problem about conclusion of field equations and motion equations of the charged splinters (the united particles with internal nuclear structure) which are generated in consequence of the chain fission reaction. These compound united particles can be considered as many time ionizated positive ions for the reason electrons upsetting of the atom outer skin of divided substance.

For definition of the electromagnetic field of the charged fission splinters we shall be proceeding in own analysis from the field microscopic equations of isolated particles (1). Add to the index $i$ the index $k$. Then instead of the vector $R_{i}$ we take the vector $R_{k i}$ and expand it into the sum of two vectors: $R_{k i}=R_{k}+r_{k i}$, where $k$ is the splinter index, $i$ is the index of the particle of given splinter, $R_{k}$ is the coordinate of the some fixed point (nucleus) of $k$-th splinter, $r_{k i}$ is the internal coordinate of $k i$-th particle (its position) concerning the fixed point of $k$-th splinter.

But as distinct from the works [2] [3] [4] and the others here it is necessary to mean that considered charged compound fission splinters aren't "the stable complexes" but are the powerfully nonsteady particles groups are exposed the pronounced instantaneous radioactive fission (the $\beta$-fission accompanied by the $\gamma$-eradiation). Radioactive instability of the fission splinters in one's turn leads to peculiar and simultaneously enough intricate deformation of the fields electromagnetic equations and the motion of these assembly particles. Thus, our problem is concluded in so that "to catch" the distinctive peculiarity of radioactive disintegration of the given instable particles and to obtain corect from mathematical and physical points of view the note of these equations.

Before to begin the equations synthesis we discuss just one more important moment of the theory. The problem of classical nonrelativistic electrodynamics is the registration of radioactive instability of the charged particles. It is well known that in the given case, i.e. when only parts up to the first order on $1 / c$ inclusive are taken into consideration, to count up the effect of radiation $\gamma$-eradiation (the fading) turn out badly. This effect caused by "minus-field" (the retarding field) of the particle in nonrelativistic case is vanishingly small. In 
connection with given exposition we try by the conclusion of equations to take into account the effect of charged radioactive disintegration but not of radioactive $\gamma$-eradiation accounting.

Subsequently it is important to distinguish between the individual charged particles in the $k$-th fission splinter in the point $R_{k i}$ with the charges $Z_{k i}$ and the charged nucleus in the point $R_{k}$ is exposed $\beta^{-}$-radioactive fission. We remind that the fission splinters are intensive $\beta^{-}$-eradiators, moreover the beta-fission is the subnucleonic process stipulated by poor interactions and proceeding for surplus neutrons of a splinter according to scheme: $n \rightarrow p+e^{-}+\tilde{v}$, i.e. in free state on average life time in order of 17 minutes neutron decays into proton, electron and antineutrino. We note also that inside of nucleus the formation of neutron it is possible owing to additional energy is imparted to proton by other nucleons of nucleus: $p \rightarrow n+e^{+} v$ i.e. during $\beta^{+}$-fission when the process of proton fission inside of nucleus is occurs at expence of nucleus energy into neutron, positron and neutrino.

Therefore, we indicate the charge in the point $R_{k}$ in the time moment $t$ through $Z_{k}$. The charge $Z_{k}$ is the total proton charge $X_{k}$ of the nucleus (initial proton charge of splinter + proton charge of $\beta^{-}$-fission products) and electron charge $Y_{k}$ of $\beta^{-}$-fission products:

$$
Z_{k}=X_{k}+Y_{k}
$$

Obviosly, value $Z_{k}$ (8) coincides with charged value of initial proton charge of splinter. Nevertheless, it is necessary to make use of expansion (8) for account of radioactive fission effect of the nucleus of $k$-th splinter.

Further we shall think that solutions $E_{*}$ and $B_{*}$ of field equations can be approximated as converged rows on parameter $\left|r_{k i}\right| /\left|R_{k}-R\right|<1$, i.e. the dimensions of a splinter $R_{k i}$ is smaller a distance $\sigma_{k}$ from the observation point $R$ to fixed point (nucleus) $R_{k}$ of $k$-th splinter. Transform equations (1) taking into account of all expansions including the $\delta$-function expansion into the Taylor's row on $r_{k i}$ in the locality of the point $\left(R_{k}-R\right)$. Then we obtain

$$
\begin{aligned}
\nabla E_{*}= & \sum_{k} \sum_{i} Z_{k i} \sum_{n=0}^{\infty} \frac{(-1)^{n}}{n !}\left(r_{k i} \nabla\right)^{n} \delta\left(R_{k}-R\right) \\
& +\sum_{k} X_{k} \delta\left(R_{k}-R\right)+\sum_{k} Y_{k} \delta\left(R_{k}-R\right), \\
-E_{*}^{\prime}+\nabla \times B_{*}= & \frac{1}{c} \sum_{k} \sum_{i} Z_{k i}\left(\dot{R}_{k}+\dot{r}_{k i}\right) \sum_{n=0}^{\infty} \frac{(-1)^{n}}{n !}\left(r_{k i} \nabla\right)^{n} \delta\left(R_{k}-R\right) \\
& +\frac{1}{c} \sum_{k} X_{k} \dot{R}_{k} \delta\left(R_{k}-R\right)+\frac{1}{c} \sum_{k} Y_{k} \dot{R}_{k} \delta\left(R_{k}-R\right), \\
& \nabla B_{*}=0, \quad B_{*}^{\prime}+\nabla \times E_{*}=0 .
\end{aligned}
$$

In equations of system (9) we have operator equality of action to threedimensional $\delta$-function:

$$
\nabla_{k} \delta\left(R_{k}-R\right)=-\nabla \delta\left(R_{k}-R\right),
$$

where $\nabla_{k}=\partial / \partial R_{k}, \nabla=\partial / \partial R$. Consider this system in detail. The first 
equation can be written in the shortened form:

$$
\nabla E_{*}=q-\nabla p+s_{+}+s_{-},
$$

where is denoted

$$
\begin{gathered}
q=\sum_{k} q_{k}, \quad q_{k}=\sum_{i} Z_{k i} \delta\left(R_{k}-R\right), \\
p=\sum_{k} p_{k}, \quad p_{k}=\sum_{i} Z_{k i} \sum_{n=1}^{\infty} \frac{(-1)^{n-1}}{n !} r_{k i}\left(r_{k i} \nabla\right)^{n-1} \delta\left(R_{k}-R\right), \\
S_{+}=\sum_{k} X_{k} \delta\left(R_{k}-R\right), \quad S_{-}=\sum_{k} Y_{k} \delta\left(R_{k}-R\right) .
\end{gathered}
$$

Then we transpose the second equation of system (9) to the form

$$
\begin{aligned}
-E_{*}^{\prime}+\nabla \times B_{*}= & \frac{1}{c}\left[j-\sum_{k} \dot{R}_{k} \nabla p_{k}+\sum_{k} \sum_{i} Z_{k i} \sum_{n=0}^{\infty} \frac{(-1)^{n}}{n !} \dot{r}_{k i}\left(r_{k i} \nabla\right)^{n} \delta\left(R_{k}-R\right)\right. \\
& \left.+\sum_{k} X_{k} \dot{R}_{k} \delta\left(R_{k}-R\right)+\sum_{k} Y_{k} \dot{R}_{k} \delta\left(R_{k}-R\right)\right],
\end{aligned}
$$

where is denoted

$$
j=\sum_{k} j_{k}, \quad j_{k}=\sum_{i} Z_{k i} \dot{R}_{k} \delta\left(R_{k}-R\right) .
$$

Further we make use of operator equality of action to the $\delta$-function:

$$
\frac{\partial}{\partial t} \delta\left(R_{k}-R\right)=-\dot{R}_{k} \nabla \delta\left(R_{k}-R\right) .
$$

Differentiate the relation (12) on $\partial / \partial(c t)$ :

$$
p_{k}^{\prime}+\frac{1}{c} \dot{R}_{k} \nabla p_{k}-\frac{1}{c} \sum_{i} Z_{k i} \sum_{n=0}^{\infty} \frac{(-1)^{n}}{(n+1) !}\left[\dot{r}_{k i} r_{k i} \nabla+n r_{k i} \dot{r}_{k i} \nabla\right]\left(r_{k i} \nabla\right)^{n-1} \delta\left(R_{k}-R\right)=0 .
$$

Then we add up relation (17) by $k$ and add to right part of Equation (14). The second equation of field (14) with aid of vector identity $\nabla \times(a \times b)=(\nabla b) a-(\nabla a) b$ justifiable for any vectors $a$ and $b$ can be written in the form

$$
-E_{*}^{\prime}+\nabla \times B_{*}=\frac{1}{c} j+p^{\prime}+\nabla \times m+\frac{1}{c}\left(u_{+}+u_{-}\right),
$$

where following designations are introduced

$$
\begin{aligned}
m=\sum_{k} m_{k}, \quad m_{k} & =\frac{1}{c}\left[p_{k} \times \dot{R}_{k}+\sum_{i} Z_{k i} \sum_{n=1}^{\infty} \frac{(-1)^{n-1}}{n(n+1) !} r_{k i} \times \dot{r}_{k i}\left(r_{k i} \nabla\right)^{n-1} \delta\left(R_{k}-R\right)\right], \\
u_{+} & =\sum_{k} X_{k} \dot{R}_{k} \delta\left(R_{k}-R\right), \quad u_{-}=\sum_{k} Y_{k} \dot{R}_{k} \delta\left(R_{k}-R\right) .
\end{aligned}
$$

Here the vector $m$ uses the name of magnetic vector.

Note that in Equations ((10), (18)) summands $s_{+}, c^{-1} u_{+}$and $s_{-}, c^{-1} u_{-}$(13), (20) can be associated with outrunning and retarding fields in relativistic and plus- and minus-fields in quantum electrodynamics [2] [15] [16] [17] [18].

Values $s_{+}, c^{-1} u_{+}$represent own fields of the charged nucleuses of all splinters and $s_{-}, C^{-1} u_{-}$represent "the deformation" of total field is caused by the effect of radioactive $\beta^{-}$-fission of nucleuses of all splinters.

We consider full charge of the $k$-th splinter 


$$
\zeta_{k}=\sum_{i} Z_{k i}+Z_{k}=\sum_{i} Z_{k i}+X_{k}+Y_{k}
$$

Taking into account designation (21) for fission-fragment charge and expressions (11) and (15) can be written that

$$
\begin{gathered}
Q=q+s_{+}+s_{-}=\sum_{k} \zeta_{k} \delta\left(R_{k}-R\right), \\
J=j+u_{+}+u_{-}=\sum_{k} \zeta_{k} \dot{R}_{k} \delta\left(R_{k}-R\right) .
\end{gathered}
$$

Values $Q$ and $J$ are determined by equalities (22) on the analogy of electrodynamic standards may call densities of the fission-fragment charge and current correspondingly.

It is easy to notice that from system (22) conservation law of the fissionfragment charge follows directly:

$$
\frac{\partial Q}{\partial t}=-\nabla J
$$

Indeed, formula (23) follows if the value $\partial Q / \partial t$ from the first relation of system (22) to calculate with the aid of equality (16) and then to make use of the second relation of system (22).

Values $p$ (12) and $m$ (19) in conformity with accepted electrodynamic standards can be named the fission-fragment electric and magnetic polarizable densities.

Thus, the system of equations for the field of charged fission splinters into terms of densities of charge, current, electric and magnetic polarization looks as:

$$
\begin{gathered}
\nabla E_{*}=Q-\nabla p, \\
-E_{*}^{\prime}+\nabla \times B_{*}=\frac{1}{c} J+p^{\prime}+\nabla \times m, \\
\nabla B_{*}=0, \quad B_{*}^{\prime}+\nabla \times E_{*}=0 .
\end{gathered}
$$

The system of Equation (24) are the system of microscopic fields $\left(E_{*}, B_{*}\right)$ induced by splinters. In other words, these fields where fission splinters are situated. Notice simultaneously that on own form these fields are quite identical to the Maxwell macroscopic field equations for continuous medium. Equation (24) can be named equations of the fission-fragment fields. If introduce instead of vectors $p$ and $m$ vectors of displacement $D_{*}$ and $H_{*}$ correspondingly to formulas

$$
D_{*}=E_{*}+p, \quad H_{*}=B_{*}-m,
$$

then obviously that the system of equations of fission-fragment fields (24) assumes the form (compare with the system of the Maxwell macroscopic equations for variable electromagnetic field $(E, D, H, B): \operatorname{rot} E=-\partial B / \partial t$, $\operatorname{div} D=\rho, \operatorname{rot} H=j+\partial D / \partial t, \operatorname{div} B=0$, where $j+\partial D / \partial t$ is the density of the full current, $j$ is the density of the conductivity current, $\rho$ is the volumetric charged density, $I=\int_{S} j \mathrm{~d} S$ is the conductivity current, $I_{*}=(\mathrm{d} / \mathrm{d} t) \int_{S} D \mathrm{~d} S$ is the displacement current, $S$ is the surface):

$$
\nabla D_{*}=Q, \quad-D_{*}^{\prime}+\nabla \times H_{*}=\frac{1}{C} J,
$$




$$
\nabla B_{*}=0, \quad B_{*}^{\prime}+\nabla \times E_{*}=0 .
$$

We now move on to formation of equations for dynamics of charged fission splinters if there are given off electrons arised as a result of radioactive $\beta$-disintegration.

The motion equation of $k i$-th particle into $k$-th splinter with the charge $Z_{k i}$, the mass $m_{k i}$ and the coordinate $R_{k i}$ at the time moment $t$ into full electromagnetic field $\left(E\left(R_{k i}, t\right), B\left(R_{k i}, t\right)\right)$ has a form of Equation (6), where $R_{i} \rightarrow R_{k i}$, i.e.

$$
m_{k i} \ddot{R}_{k i}=Z_{k i}\left[E\left(R_{k i}, t\right)+\frac{1}{c} \dot{R}_{k i} \times B\left(R_{k i}, t\right)\right],
$$

moreover full fields $E\left(R_{k i}, t\right)$ and $B\left(R_{k i}, t\right)$ in considered case (compare with expressions for $E$ and $B$ in Equation (7)) have a form

$$
\begin{aligned}
E\left(R_{k i}, t\right)= & -\sum_{j} \nabla_{k i} \frac{Z_{k j}}{4 \pi \sigma_{k l, i j}}-\nabla_{k i} \frac{\left(X_{k}+Y_{k}\right)}{4 \pi \sigma_{k, k}}-\sum_{l} \sum_{j} \nabla_{k i} \frac{Z_{l j}}{4 \pi \sigma_{k l, i j}} \\
& -\sum_{l} \nabla_{k i} \frac{\left(X_{l}+Y_{l}\right)}{4 \pi \sigma_{k l, i l}}+E_{0}\left(R_{k i}, t\right), \\
B\left(R_{k i}, t\right)= & B_{0}\left(R_{k i}, t\right),
\end{aligned}
$$

where $j \neq i, l \neq k ; \nabla_{k i}$-is the gradient vector on the elements of vector $R_{k i}$. In the system of Equation (27) are denoted

$$
\begin{gathered}
\sigma_{k, i j}=\left|R_{k i}-R_{k j}\right|, \quad \sigma_{k, i k}=\left|R_{k i}-R_{k k}\right|=\left|R_{k i}-R_{k}\right|, \\
\sigma_{k l, i j}=\left|R_{k i}-R_{l j}\right|, \quad \sigma_{k l, i l}=\left|R_{k i}-R_{l l}\right|=\left|R_{k i}-R_{l}\right|, \\
R_{k k} \equiv R_{k}, \quad R_{l l} \equiv R_{l}, \quad\left|R_{k i}-R_{k}\right|=\left|r_{k i}\right| .
\end{gathered}
$$

One can see beautifully from relations (27) that full field $(E, B)$ includes:

1) intrafission-fragment field created by all particles $k i, i \neq j$, of given $k$-th splinter and by nucleus of this splinter;

2) interfission-fragment fields corresponding to particles of all other $l$ splinters, $l \neq k$, and to nucleuses of these splinters also;

3) external field $\left(E_{0}, B_{0}\right)$ created by external sources.

On the analogy of (6) $(i \rightarrow k)$ can be written the motion equation of the nucleus of $k$-th splinter with charge $Z_{k}=X_{k}+Y_{k}$, mass $m_{k}$, coordinate $R_{k}$ into full electromagnetic field $\left(E\left(R_{k}, t\right), B\left(R_{k}, t\right)\right)$ :

$$
m_{k} \ddot{R}_{k}=Z_{k}\left[E\left(R_{k}, t\right)+\frac{1}{c} \dot{R}_{k} \times B\left(R_{k}, t\right)\right],
$$

where

$$
\begin{aligned}
E\left(R_{k}, t\right)= & -\sum_{j} \nabla_{k} \frac{Z_{k j}}{4 \pi \sigma_{k, k j}}-\sum_{l} \sum_{j} \nabla_{k} \frac{Z_{l j}}{4 \pi \sigma_{k l, k j}} \\
& -\sum_{l} \nabla_{k} \frac{\left(X_{l}+Y_{l}\right)}{4 \pi \sigma_{k l, k l}}+E_{0}\left(R_{k}, t\right), \\
B\left(R_{k}, t\right)= & B_{0}\left(R_{k}, t\right),
\end{aligned}
$$

for $l \neq k$, i.e. system of relations (29), (30) can be obtained from system (26), 
(27) by $i=k, \nabla_{k k} \equiv \nabla_{k}$ and taking into account identical equalities (28). In this case we think in relation (27) that the second summand in right part is equal to zero, $\nabla_{k}=\partial / \partial R_{k}$.

After summarizing of Equation (26) on $i$ and adding with Equation (29) for description of the motion of $k$-th splinter with mass barm $_{k}$ and coordinate of masses center $\bar{R}_{k}$ :

$$
\bar{m}_{k}=\sum_{k i}+m_{k}, \quad \bar{R}_{k}=\sum_{i} \frac{m_{k i} R_{k i}+m_{k} R_{k}}{\bar{m}_{k}},
$$

we obtain the motion equation of $k$-th splinter in the form

$$
\bar{m}_{k} \ddot{\bar{R}}_{k}=\sum_{i} Z_{k i}\left[E\left(R_{k i}, t\right)+\frac{1}{c} \dot{R}_{k i} \times B\left(R_{k i}, t\right)\right]+Z_{k}\left[E\left(R_{k}, t\right)+\frac{1}{c} \dot{R}_{k} \times B\left(R_{k}, t\right)\right] .
$$

Substitute expressions (27), (30) into Equation (31). Then resultant force corresponding to intrafission-fragment field for the set of central forces is obviously equal zero. Consequently Equation (31) acquires the following form:

$$
\begin{aligned}
\bar{m}_{k} \ddot{\bar{R}}_{k}= & -\sum_{l} \sum_{i} \sum_{j} \nabla_{k i} \frac{Z_{k i} Z_{l j}}{4 \pi \sigma_{k l, j j}}-\sum_{l} \sum_{i} \nabla_{k i} \frac{Z_{k i}\left(X_{l}+Y_{l}\right)}{4 \pi \sigma_{k i, i l}} \\
& -\sum_{l} \sum_{j} \nabla_{k} \frac{Z_{l j}\left(X_{k}+Y_{k}\right)}{4 \pi \sigma_{k l, k j}}-\sum_{l} \nabla_{k} \frac{\left(X_{l}+Y_{l}\right)\left(X_{k}+Y_{k}\right)}{4 \pi \sigma_{k l, k l}} \\
& +\sum_{i} Z_{k i}\left[E_{0}\left(R_{k i}, t\right)+\frac{1}{C} \dot{R}_{k i} \times B_{0}\left(R_{k i}, t\right)\right] \\
& +\left(X_{k}+Y_{k}\right)\left[E_{0}\left(R_{k}, t\right)+\frac{1}{C} \dot{R}_{k} \times B_{0}\left(R_{k}, t\right)\right],
\end{aligned}
$$

where $l \neq k$, and external field $\left(E_{0}, B_{0}\right)$ satisfies to homogeneous equations

$$
\nabla B_{0}=0, \quad B_{0}^{\prime}+\nabla \times E_{0}=0 .
$$

Thus, Equation (32) is the motion equation of $k$-th splinter into electromagnetic field of other splinters and external sources.

It is important to note that Equation (32) on a level with traditional summands in the right part of the Lorenz force contains also summands are made a force connected with radioactive charged eradiation of nucleuses into fission splinters (the accounting of $\beta^{-}$-charged eradiation by the radioactive electrodynamic effect).

In following section will be realized the breeding of "the energy equation" for fission splinters with charged radioactivity, namely the equation in conformity with it total derivative on the time from energy of system is equal to short-active and long-active contributions containing the powerful components of different forces.

\section{Power Relations for the Charged Fission Splinters}

Proceed to the substantiation of energy equation and internal pulse moment of $k$-th splinter on the basis of previous section results.

For working out of energy equation we take $k i$-th particle and scalarly multiply motion Equation (26) by velocity $\dot{R}_{k i}$ : 


$$
m_{k i} \dot{R}_{k i} \ddot{R}_{k i}=Z_{k i} \dot{R}_{k i} E\left(R_{k i}, t\right) .
$$

Similarly we treat with the nucleus of $k$-th splinter. From Equation (29) after multiply by the vector $\dot{R}_{k}$ we have

$$
m_{k} \dot{R}_{k} \ddot{R}_{k}=Z_{k} \dot{R}_{k} E\left(R_{k}, t\right) .
$$

Then summarise by $i$ Equation (33) and add with Equation (34). With the aid of expressions for $\bar{m}_{k}, \bar{R}_{k}$ and of relations (27), (30) we find for internal coordinate $\bar{r}_{k i}=R_{k i}-\bar{R}_{k}$ that

$$
\begin{aligned}
& \frac{\mathrm{d}}{\mathrm{d} t}\left(\frac{1}{2} \sum_{i} m_{k i} \dot{R}_{k i}^{2}+\frac{1}{2} m_{k} \dot{R}_{k}^{2}\right) \\
& =\sum_{i} Z_{k i} \dot{R}_{k i} E\left(R_{k i}, t\right)+Z_{k} \dot{R}_{k} E\left(R_{k}, t\right),
\end{aligned}
$$

or

$$
\begin{aligned}
& \frac{\mathrm{d}}{\mathrm{d} t}\left(\frac{1}{2} \sum_{i} m_{k i} \dot{\bar{r}}_{k i}^{2}+\sum_{i} m_{k i} \dot{\bar{r}}_{k i} \dot{\bar{R}}_{k}+\frac{1}{2} \bar{m}_{k} \dot{\bar{R}}_{k}^{2}+\frac{1}{2} m_{k}\left(\dot{R}_{k}^{2}-\dot{\bar{R}}_{k}^{2}\right)\right) \\
& =-\sum_{i, j} \dot{R}_{k i} \nabla_{k i} \frac{Z_{k i} Z_{k j}}{4 \pi \sigma_{k, i j}}-\sum_{i} \dot{R}_{k i} \frac{\nabla_{k i} Z_{k i} Z_{k}}{4 \pi \sigma_{k, i k}} \\
& \quad-\sum_{l, i, j} \dot{R}_{k i} \nabla_{k i} \frac{Z_{k i} Z_{l j}}{4 \pi \sigma_{k l, i j}}-\sum_{l, i} \dot{R}_{k i} \nabla_{k i} \frac{Z_{k i}\left(X_{l}+Y_{l}\right)}{4 \pi \sigma_{k l, i l}} \\
& \quad+\sum_{i} \dot{R}_{k i} Z_{k i} E_{0}\left(R_{k i}, t\right)-\sum_{j} \dot{R}_{k} \nabla_{k} \frac{Z_{k} Z_{k j}}{4 \pi \sigma_{k, k j}}-\sum_{l, j} \dot{R}_{k} \nabla_{k} \frac{\left(X_{k}+Y_{k}\right) Z_{l j}}{4 \pi \sigma_{l k, k j}} \\
& \quad-\sum_{l} \dot{R}_{k} \nabla_{k} \frac{\left(X_{k}+Y_{k}\right)\left(X_{l}+Y_{l}\right)}{4 \pi \sigma_{k l, k l}}+\dot{R}_{k}\left(X_{k}+Y_{k}\right) E_{0}\left(R_{k}, t\right),
\end{aligned}
$$

where $i \neq j, l \neq k$.

In right part of Equation (35) the first, the second and the sixth summands are making intrafission-fragment power contribution which can be presented as a total derivative on the time from intrafission-fragment Coulomb's energy of given $k$-th splinter $E_{C, k}$ :

$$
\begin{aligned}
& -\sum_{i, j} \dot{R}_{k i} \nabla_{k i} \frac{Z_{k i} Z_{k j}}{4 \pi \sigma_{k, i j}}-\sum_{i} \dot{R}_{k i} \nabla_{k i} \frac{Z_{k i} Z_{k}}{4 \pi \sigma_{k, i k}}-\sum_{j} \dot{R}_{k} \nabla_{k} \frac{Z_{k} Z_{k j}}{4 \pi \sigma_{k, k j}} \\
= & -\sum_{i, j}\left(\dot{R}_{k i} \nabla_{k i}+\dot{R}_{k j} \nabla_{k j}\right) \frac{Z_{k i} Z_{k j}}{8 \pi \sigma_{k, i j}}-\sum_{i}\left(\dot{R}_{k i} \nabla_{k i}+\dot{R}_{k} \nabla_{k}\right) \frac{Z_{k i} Z_{k}}{8 \pi \sigma_{k, i k}} \\
& -\sum_{j}\left(\dot{R}_{k} \nabla_{k}+\dot{R}_{k j} \nabla_{k j}\right) \frac{Z_{k} Z_{k j}}{8 \pi \sigma_{k, k j}} \\
= & -\frac{\mathrm{d}}{\mathrm{d} t} E_{C, k},
\end{aligned}
$$

or

$$
\frac{\mathrm{d}}{\mathrm{d} t} E_{C, k}=\frac{\mathrm{d}}{\mathrm{d} t} \frac{1}{8 \pi}\left\{\sum_{i, j} \frac{Z_{k i} Z_{k j}}{\left|\bar{r}_{k i}-\bar{r}_{k j}\right|}+\sum_{i} \frac{Z_{k i} Z_{k}}{\left|\bar{r}_{k i}+\bar{R}_{k}-R_{k}\right|}+\sum_{j} \frac{Z_{k} Z_{k j}}{\left|\bar{r}_{k j}+\bar{R}_{k}-R_{k}\right|}\right\} .
$$

In general case Equation (35) taking into account the dependence (36) can be produced in the form 


$$
\begin{aligned}
& \frac{\mathrm{d}}{\mathrm{d} t}\left(\frac{1}{2} \sum_{i} m_{k i} \dot{\bar{r}}_{k i}^{2}+\sum_{i} m_{k i} \dot{\dot{\bar{r}}_{k i}} \dot{\bar{R}_{k}}+\frac{1}{2} \bar{m}_{k} \dot{\bar{r}}_{k}^{2}+\frac{1}{2} m_{k}\left(\dot{R}_{k}^{2}-\dot{\bar{R}}_{k}^{2}\right)+E_{C, k}\right) \\
& =\Lambda_{k, l}+\Lambda_{k, 0},
\end{aligned}
$$

where is denoted

$$
\begin{aligned}
\Lambda_{k, l}= & -\sum_{l, i, j}\left(\dot{\bar{R}}_{k}+\dot{\bar{r}}_{k i}\right) \nabla_{k i} \frac{Z_{k k} Z_{l j}}{4 \pi \sigma_{k l, i j}}-\sum_{l, i}\left(\dot{\bar{R}}_{k}+\dot{\bar{r}}_{k i}\right) \nabla_{k i} \frac{Z_{k i}\left(X_{l}+Y_{l}\right)}{4 \pi \sigma_{k l, i l}} \\
& -\sum_{l, j} \dot{R}_{k} \nabla_{k} \frac{\left(X_{k}+Y_{k}\right) Z_{l j}}{4 \pi \sigma_{k l, k j}}-\sum_{l} \dot{R}_{k} \nabla_{k} \frac{\left(X_{k}+Y_{k}\right)\left(X_{l}+Y_{l}\right)}{4 \pi \sigma_{k l, k l}}, \\
\Lambda_{k, 0} & =\sum_{i}\left(\dot{\bar{R}}_{k}+\dot{\bar{r}}_{k i}\right) Z_{k i} E_{0}\left(R_{k i}, t\right)+\dot{R}_{k}\left(X_{k}+Y_{k}\right) E_{0}\left(R_{k}, t\right) .
\end{aligned}
$$

Thus, the energy equation of $k$-th splinter (37) in own left part contains summands of intrafission-fragment kinetic, potential and Coulomb's energies; quantities determining interfission-fragment $\Lambda_{k, l}$ and external $\Lambda_{k, 0}$ fields on the background of $\beta^{-}$-charged eradiation were in the right part of this equation.

For working out of internal pulse moment equation of $k$-th splinter we make use of corresponding expression for the quantity of internal pulse moment

$$
\Psi_{k}=\sum_{i} m_{k i} \bar{r}_{k i} \times \dot{\bar{r}}_{k i}+m_{k}\left(R_{k}-\bar{R}_{k}\right) \times\left(\dot{R}_{k}-\dot{\bar{R}}_{k}\right),
$$

where $m_{k i}, m_{k}$ are masses of composed particles and nucleus of $k$-th splinter, $\bar{r}_{k i}=R_{k i}-\bar{R}_{k}$ are internal coordinates of particles.

Differentiate relation (38) on the time. Then we obtain with employment of significances of $\bar{m}_{k}, \bar{R}_{k}$ :

$$
\begin{aligned}
\dot{\Psi}_{k} & =\sum_{i} m_{k i} \bar{r}_{k i} \times \ddot{\bar{r}}_{k i}+m_{k}\left(R_{k}-\bar{R}_{k}\right) \times\left(\ddot{R}_{k}-\ddot{\bar{R}}_{k}\right) \\
& =\sum_{i} m_{k i} \bar{r}_{k i} \times \ddot{R}_{k i}-m_{k}\left(\bar{R}_{k}-R_{k}\right) \times \ddot{\bar{R}}_{k}+m_{k}\left(R_{k}-\bar{R}_{k}\right) \times\left(\ddot{R}_{k}-\ddot{\bar{R}}_{k}\right) \\
& =\sum_{i} m_{k i} \bar{r}_{k i} \times \ddot{R}_{k i}+m_{k}\left(R_{k}-\bar{R}_{k}\right) \times \ddot{R}_{k} .
\end{aligned}
$$

The following step: put motion Equations ((26), (29)) and equations of fields (27), (30) into Equation (39). We obtain finally

$$
\begin{aligned}
\dot{\Psi}_{k}= & -\sum_{l, i, j} \bar{r}_{k i} \times \nabla_{k i} \frac{Z_{k i} Z_{l j}}{4 \pi \sigma_{k l, i j}}-\sum_{l, i} \bar{r}_{k i} \times \nabla_{k i} \frac{Z_{k i}\left(X_{l}+Y_{l}\right)}{4 \pi \sigma_{k l, i l}} \\
= & \sum_{i} Z_{k i} \bar{r}_{k i} \times\left[E_{0}\left(R_{k i}, t\right)+\frac{1}{c} \dot{R}_{k i} \times B_{0}\left(R_{k i}, t\right)\right] \\
& -\sum_{l, j}\left(R_{k}-\bar{R}_{k}\right) \times \nabla_{k} \frac{\left(X_{l}+Y_{k}\right) Z_{l j}}{4 \pi \sigma_{k l, k j}} \\
& -\sum_{l}\left(R_{k}-\bar{R}_{k}\right) \times \nabla_{k} \frac{\left(X_{k}+Y_{k}\right)\left(X_{l}+Y_{l}\right)}{4 \pi \sigma_{k l, k l}} \\
& +\left(X_{k}+Y_{k}\right)\left(R_{k}-\bar{R}_{k}\right) \times\left[E_{0}\left(R_{k}, t\right)+\frac{1}{C} \dot{R}_{k} \times B_{0}\left(R_{k}, t\right)\right],
\end{aligned}
$$

where $i \neq j, l \neq k$ and resulting activity of central forces corresponding intrafission-fragment fields is equal zero. 


\section{Statistical Description of Nonrelativistic Nuclear Electrodynamics Laws}

Maxwell's equations of electromagnetic field are in reality macroscopic equations for the description of field and substance with slowly-varying (into time and space) physical variables. Consequently their employment for the description of microscopic objects behaviour with quicly-varying physical variables cannot be recognized fully adequate and theoretically corect.

Macroscopic equations operate with not separate particle but with whole group of particles (medium). Obviously from this point of view that for the obtaining of nuclear electromagnetic macroscopic laws it is necessary to carry out averaging on fission splinters and to avail of statistical mechanics principles where physical variables describing with the aid of distribution functions are considered as continuous functions of spatio-temporal coordinates.

We note also for the carrying-out of statistical (macroscopic) physics it is necessary that averaging on considered splinters occured in the volume with sizes bigger than interfission-fragment distances but smaller than macroscopic sizes of the system.

Statistical averaging in statistical mechanics is introduced with the aid of distribution function or phase density $f(x)$ in phase space where $x(t)$ is phase vector of the system [19] [20].

In nonrelativistic case macroscopic values can be averaged only on phase space of coordinates and velocities of particles. Therefore, if $k i$-th particle is characterized by microscopic value $a\left(R_{k i}, \dot{R}_{k i}, t\right)$ then the average from it is defined by this integral

$$
\mathcal{A}(R, t)=\langle a\rangle=\int a f \mathrm{~d} \varphi,
$$

where $f=f\left(R_{k i}, \dot{R}_{k i}, t\right)$ is distribution function, $\mathrm{d} \varphi=\prod_{k i} \mathrm{~d} R_{k i} \mathrm{~d} \dot{R}_{k i} \quad$ is a element of the phase space volume, $f \mathrm{~d} \varphi$ is the probability of system stay in the element of volume $\mathrm{d} \varphi$ (owing to the conservation of probability $f \mathrm{~d} \varphi=$ const along phase trajectory).

Hence it follows the commutation of operations of the differentiation on the time and the averaging on phase space and spatial differentiation and phase averaging also:

$$
\begin{gathered}
\frac{\partial}{\partial t}\langle a\rangle=\frac{\partial}{\partial t} \int a f \mathrm{~d} \varphi=\int \frac{\mathrm{d} a}{\mathrm{~d} t} f \mathrm{~d} \varphi=\left\langle\frac{\mathrm{d} a}{\mathrm{~d} t}\right\rangle, \\
\nabla\langle a\rangle=\nabla \int a f \mathrm{~d} \varphi=\int \nabla a f \mathrm{~d} \varphi=\langle\nabla a\rangle,
\end{gathered}
$$

where

$$
\begin{gathered}
\frac{\mathrm{d}}{\mathrm{d} t}=\frac{\partial}{\partial t}+\sum_{k i} \dot{R}_{k i} \nabla_{k i}+\sum_{k i} \ddot{R}_{k i} \nabla_{\cdot, k i}, \\
\nabla_{k i} \equiv \frac{\partial}{\partial R_{k i}}, \quad \nabla_{\cdot, k i} \equiv \frac{\partial}{\partial \dot{R}_{k i}} .
\end{gathered}
$$

So that to receive Maxwell's equations it is necessary to apply statistical averaging to equations of fission-fragment fields (24). Then we shall be having 


$$
\begin{gathered}
\left\langle\nabla E_{*}\right\rangle=\langle Q\rangle-\langle\nabla p\rangle, \\
-\left\langle E_{*}^{\prime}\right\rangle+\left\langle\nabla \times B_{*}\right\rangle=\frac{1}{c}\langle J\rangle+\left\langle p^{\prime}\right\rangle+\langle\nabla \times m\rangle, \\
\left\langle\nabla B_{*}\right\rangle=0, \quad\left\langle B_{*}^{\prime}\right\rangle+\left\langle\nabla \times E_{*}\right\rangle=0,
\end{gathered}
$$

and further, with the aid of commutation rule (41) in system of Equation (42) we obtain

$$
\begin{gathered}
\nabla\left\langle E_{*}\right\rangle=\langle Q\rangle-\nabla\langle p\rangle, \\
-\left\langle E_{*}\right\rangle^{\prime}+\nabla \times\left\langle B_{*}\right\rangle=\frac{1}{c}\langle J\rangle+\langle p\rangle^{\prime}+\nabla \times\langle m\rangle, \\
\nabla\left\langle B_{*}\right\rangle=0, \quad\left\langle B_{*}\right\rangle^{\prime}+\nabla \times\left\langle E_{*}\right\rangle=0 .
\end{gathered}
$$

Use designation (40) for macroscopic fields, densities of charge, current and vectors of polarizations

$$
\begin{array}{cl}
\mathcal{E}=\left\langle E_{*}\right\rangle, \quad \mathcal{B}=\left\langle B_{*}\right\rangle, \quad \mathcal{Q}=\langle Q\rangle, \quad \mathcal{J}=\langle J\rangle, \\
\mathcal{P}=\langle p\rangle, \quad \mathcal{M}=\langle m\rangle .
\end{array}
$$

Then we can rewrite system (43) in the form of Maxwell's equations:

$$
\begin{gathered}
\nabla \mathcal{E}=\mathcal{Q}-\nabla \mathcal{P}, \\
-\mathcal{E}^{\prime}+\nabla \times \mathcal{B}=\frac{1}{c} \mathcal{J}+\mathcal{P}^{\prime}+\nabla \times \mathcal{M}, \\
\nabla \mathcal{B}=0, \quad \mathcal{B}^{\prime}+\nabla \times \mathcal{E}=0 .
\end{gathered}
$$

If resort to designations for vectors of displacement $(\mathcal{D}=\mathcal{E}+\mathcal{P}, \mathcal{H}=\mathcal{B}-\mathcal{M})$, system of Maxwell's Equation (44) can be written in still more compact form:

$$
\begin{gathered}
\nabla \mathcal{D}=\mathcal{Q}, \quad-\mathcal{D}^{\prime}+\nabla \times \mathcal{H}=\frac{1}{C} \mathcal{J}, \\
\nabla \mathcal{B}=0, \quad \mathcal{B}^{\prime}+\nabla \times \mathcal{E}=0 .
\end{gathered}
$$

System of Equation (45) can be obtained by means of the averaging of system (25) also. We note that system of Equation (45) into known terms of Maxwell fields $\mathcal{E}, \mathcal{B}, \mathcal{D}$ and $\mathcal{H}$ is comfortable for the employment in the case of assignment of some boundary conditions.

Continue the derivation of macroscopic equations on the basis of the averaging of microscopic equations. Then analogously by means of the averaging of Equation (23) can obtain macroscopic equation of the charge conservation

$$
\frac{\partial \mathcal{Q}}{\partial t}=-\nabla \mathcal{J}
$$

If further to average relations (22) then we find macroscopic densities of charge and current correspondingly:

$$
\mathcal{Q}(R, t)=\langle Q\rangle=\left\langle\sum_{k} \zeta_{k} \delta\left(R_{k}-R\right)\right\rangle,
$$




$$
\mathcal{J}(R, t)=\langle J\rangle=\left\langle\sum_{k} \zeta_{k} \dot{R}_{k} \delta\left(R_{k}-R\right)\right\rangle .
$$

Write solutions of macroscopic Equation (44) to an approximation of summands in order $1 / c$ :

$$
\begin{gathered}
\mathcal{E}(R, t)=\mathcal{E}_{0}(R, t)-\nabla \int\left[\mathcal{Q}\left(R_{\alpha}, t\right)-\nabla_{\alpha} \mathcal{P}\left(R_{\alpha}, t\right)\right] \frac{1}{4 \pi \sigma_{\alpha}} \mathrm{d} R_{\alpha}, \\
\mathcal{B}(R, t)=\mathcal{B}_{0}(R, t)+\nabla \times \int\left[\frac{1}{c} \mathcal{J}\left(R_{\alpha}, t\right)+\frac{1}{c} \frac{\partial \mathcal{P}\left(R_{\alpha}, t\right)}{\partial t}+\nabla_{\alpha} \times \mathcal{M}\left(R_{\alpha}, t\right)\right] \frac{1}{4 \pi \sigma_{\alpha}} \mathrm{d} R_{\alpha},
\end{gathered}
$$

where $\nabla_{\alpha}=\partial / \partial R_{\alpha}, \sigma_{\alpha}=\left|R-R_{\alpha}\right|,\left(\mathcal{E}_{0}, \mathcal{B}_{0}\right)$ is the solution of field equations without sources (external field). For the checking of these expressions it is enough to put their in equations (44) and to take into account dependence (46).

\section{Quantumomechanical Model of Microscopic Equations}

Here we try to apply received earlier results for microscopic equations of nuclear electrodynamics in nonrelativistic approximation to the model founded on quantum mechanics with employment of operators theory and own vectors of states in Hilbert's space.

Let there is the system of particles with masses $m_{i}$, charges $Z_{i}$ which is described by operators of coordinate $\hat{R}_{i}$ and pulse $\hat{P}_{i}$ into external electromagnetic field with potentials $\left(\varphi_{0}, A_{0}\right)$ with the aid of Hermite (since the energy is real value) operator of Hamilton to an approximation of summands in order $1 / c$ :

$$
\hat{H}=\sum_{i} \frac{\hat{P}_{i}}{2 m_{i}}+\sum_{i} \sum_{j} \frac{Z_{i} Z_{j}}{8 \pi \hat{\sigma}_{i j}}+\sum_{i} Z_{i}\left[\varphi_{0}\left(\hat{R}_{i}, t\right)-\frac{1}{2 c}\left\{\hat{P}_{i} m_{i}, A_{0}\left(\hat{R}_{i}, t\right)\right\}\right],
$$

where $\hat{\sigma}_{i j}=\left|\hat{R}_{i}-\hat{R}_{j}\right|$, the anti-commutator by scalar product of pulse operator $\hat{P}_{i}$ and vector potential $A_{0}$ is defined on the rule $\{A, B\}=A B+B A$ Operator $\hat{H}$ (47) gives the behaviour in the time of state vector $\psi(t)$ for considered system according to Schrödinger's equation:

$$
\hat{H} \psi(t)=-\frac{\hbar}{i} \frac{\partial}{\partial t} \psi(t) .
$$

Here $i$ is the imaginary unit.

Field equations into terms of Hermite's operators of electric and magnetic fields have the form

$$
\begin{gathered}
\nabla \hat{E}=\sum_{i} Z_{i} \delta\left(\hat{R}_{i}-R\right), \\
-\hat{E}^{\prime}+\nabla \times \hat{B}=\frac{1}{2 c} \sum_{i}\left\{\dot{\hat{R}}_{i}, \delta\left(\hat{R}_{i}-R\right)\right\}, \\
\nabla \hat{B}=0, \quad \hat{B}^{\prime}+\nabla \times \hat{E}=0,
\end{gathered}
$$

where in the second equation of system (49) in right part is the anti-commutator providing the hermicity of sources in field equations and what's more the relation

$$
\dot{\hat{A}}=c \hat{A}^{\prime}=\frac{i}{\hbar}[\hat{H}, \hat{A}]+\frac{\partial \hat{A}}{\partial t}
$$


has place for the operator $\hat{A}$. In equality (50) $\partial \hat{A} / \partial t$ means the open derivative on the time, i.e. we assume that the operator $\hat{A}$ depends from the time obviously. Specifically, if in expression (50) the operator $\hat{A}$ don't depends from the time obviosly then we have known as Heisenberg's motion equation for operators which are independent from the time obviosly [21] [22]:

$$
\frac{\mathrm{d} \hat{A}(t)}{\mathrm{d} t}=\frac{i}{\hbar}[\hat{H}(t), \hat{A}(t)] .
$$

Since Equation (51) has open similarity with corresponding equation of classical mechanics of Poisson's brackets then the commutator in the right part of Equations ((50), (51)) is named quantum brackets of Poisson from $\hat{H}$ and $\hat{A}$ also. We remind that commutator by two operators is named the moved form $[A, B]=A B-B A$. We note also that $\dot{\hat{R}}_{i}=\hat{P}_{i} / m_{i}$ follows from expression (47) with an approximation of summands in order $c^{0}$.

So that to write motion equation for charged $i$-th particle it is necessary by the calculation of derivatives on the time from $\hat{R}_{i}$ to take advantage of rule (50). Then with the aid of expression for $\hat{H}$ (47) we obtain

$$
\begin{gathered}
m_{i} \dot{\hat{R}}_{i}=\hat{P}_{i}-\frac{1}{c} Z_{i} A_{0}\left(\hat{R}_{i}, t\right), \\
m_{i} \ddot{\hat{R}}_{i}=-\hat{\nabla}_{i} \sum_{j} \frac{Z_{i} Z_{j}}{4 \pi \hat{\sigma}_{i j}}+Z_{i}\left[E_{0}\left(\hat{R}_{i}, t\right)+\frac{1}{2 c}\left\{\frac{\hat{P}_{i}}{m_{i}}, B_{0}\left(\hat{R}_{i}, t\right)\right\}\right],
\end{gathered}
$$

where $\hat{\nabla}_{i}=\partial / \partial \hat{R}_{i}, i \neq j$. Here external field $\left(E_{0}, B_{0}\right)$ is connected with scalar and vector potentials $\left(\varphi_{0}, A_{0}\right)$ by dependences (2), (3) so that

$$
E_{0}=-\nabla \varphi_{0}-A_{0}^{\prime}, \quad B_{0}=\nabla \times A_{0},
$$

moreover in the right part of Equation (52) over the $\{A, B\}^{\times}$is indicated rearranged form with vector product: $\{A, B\}^{\times}=A \times B-B \times A$.

In conclusion we adduce quantumomechanical description of microscopic field equations of charged fission splinters (24) for average values of corresponding operators. The system in Hilbert's space is described by the state vector $\psi(t)$ or Hermite's operator of the density $\hat{\Psi}(t)=\psi(t) \psi^{*}(t)$. Average values $\bar{A}$ of the operator $\hat{A}$ is introduced as

$$
\bar{A}=\psi^{*} \hat{A} \psi \text { or } \bar{A}=\operatorname{Sp}(\hat{\Psi} \hat{A}) .
$$

Formally for notes of fields equations of fission splinters it is necessary demand the performance of the condition $\left|r_{k i}\right|=\left|R_{k i}-R_{k}\right|<\left|R_{k}-R\right|$ which guarantees the participation of those observation points $R$ situated out of splinters and which provides the convergence of the row expansion (in this case the row converges for sources).

We have for average values of operators (compare with system (24)):

$$
\begin{gathered}
\nabla \bar{E}=\bar{Q}-\nabla \bar{p}, \\
-\bar{E}^{\prime}+\nabla \times \bar{B}=\frac{1}{c} \bar{J}+\bar{p}^{\prime}+\nabla \times \bar{m},
\end{gathered}
$$




$$
\nabla \bar{B}=0, \quad \bar{B}^{\prime}+\nabla \times \bar{E}=0,
$$

where operators are determined by expressions

$$
\begin{gathered}
\hat{Q}=\sum_{k} \zeta_{k} \delta\left(\hat{R}_{k}-R\right), \\
\hat{J}=\frac{1}{2} \sum_{k} \zeta_{k}\left\{\hat{v}_{k}, \delta\left(\hat{R}_{k}-R\right)\right\}, \quad \hat{v}_{k}=\frac{i}{\hbar}\left[\hat{H}, \hat{R}_{k}\right],
\end{gathered}
$$

and the anti-commutator and the commutator for velocity operator are in the second and the third relations (54) correspondingly.

Motion equation of $k i$-th particle in $k$-th splinter has the form of operator equation:

$$
m_{k i} \hat{R}_{k i}=Z_{k i}\left[\hat{E}\left(\hat{R}_{k i}, t\right)+\frac{1}{2 c}\left\{\hat{P}_{k i} m_{k i}, B_{0}\left(\hat{R}_{k i}, t\right)\right\}^{\times}\right]
$$

where

$$
\begin{aligned}
& \hat{E}\left(\hat{R}_{k i}, t\right)= \frac{1}{4 \pi} \hat{\nabla}_{k i}\left[-\sum_{j} Z_{k j} \hat{\sigma}_{k, i j}-\left(X_{k}+Y_{k}\right) \hat{\sigma}_{k, i k}-\sum_{l} \sum_{j} Z_{l j} \hat{\sigma}_{k l, i j}\right. \\
&\left.-\sum_{l}\left(X_{l}+Y_{l}\right) \hat{\sigma}_{k l, i l}\right]+E_{0}\left(\hat{R}_{k i}, t\right), \\
& \hat{B}\left(\hat{R}_{k i}, t\right)=B_{0}\left(\hat{R}_{k i}, t\right) ; j \neq i, l \neq k, \hat{\nabla}_{k i}=\frac{\partial}{\partial \hat{R}_{k i}} .
\end{aligned}
$$

Here the designation $\hat{\sigma}$ indicates only on the presence of coordinates operator $\hat{R}$ with corresponding indexes.

Analogously the motion equation of nucleus of $k$-th splinter can be written in the operator form:

$$
m_{k} \ddot{\hat{R}}_{k}=Z_{k}\left[\hat{E}\left(\hat{R}_{k}, t\right)+\frac{1}{2 c}\left\{\frac{\hat{P}_{k}}{m_{k}}, B_{0}\left(\hat{R}_{k}, t\right)\right\}^{\times}\right],
$$

where

$$
\begin{aligned}
& \hat{E}\left(\hat{R}_{k}, t\right)=\frac{1}{4 \pi} \hat{\nabla}_{k}-\left[\sum_{j} Z_{k j} \hat{\sigma}_{k, k j}-\sum_{l} \sum_{j} Z_{l j} \hat{\sigma}_{k l, k j}-\sum_{l}\left(X_{l}+Y_{l}\right) \hat{\sigma}_{k l, k l}\right]+E_{0}\left(\hat{R}_{k}, t\right), \\
& \hat{B}\left(\hat{R}_{k}, t\right)=B_{0}\left(\hat{R}_{k}, t\right) .
\end{aligned}
$$

Summation of motion equations of particles (55) and addition their with motion equation of nucleus (56) as a result led to a motion equation of the masses center of $k$-th splinter.

Field Equations ((49), (53)) and motion equations of charged particles and fission splinters (52), (55), (56) form the base for further deduction of operator macroscopic field equations and motion equations into quantumomechanical interpretation taking into account $\beta^{-}$-charged radioactive eradiation.

\section{Conclusion}

Here in essence the nuclear electrodynamics theory is presented. This theory opens wide perspectives for the creation of power devices in the form of the 
nuclear electrogenerator which functions on new principles of the passing of nuclear fission reaction into directional electromagnetic fields. Chain fission of heavy nuclei is one of many physical phenomena which proceed in an avalanche scheme. In fission there is an impetuous increase in the numbers of neutrons, charged particles and fission splinters with enormous kinetic energy. The present theory aims to describe the above process quantitatively. In the future it could find an application in powerful nuclear electrogenerators operating exclusively on the basis of electronuclear conversions.

\section{References}

[1] Tertychny-Dauri, V.Yu. (2015) Effect of $\beta^{-}$-Charged Eradiation and Its Calculation in the Nuclear Electrodynamics Theory. IV Int. Conf. on Mathematical Modeling in Physical Sciences (5-8.06.2015, Mykonos, Greece). IOP Journal of Physics: Conf. Series, 633, 012037.

[2] de Groot, S.R. and Suttorp, L.G. (1972) Foundations of Electrodynamics. NorthHolland Publishing Company, Amsterdam.

[3] Griffiths, D.J. (2012) Introduction to Electrodynamics. Pearson Education, London.

[4] Artsimovich, L.A. and Lukyanov, S.Yu. (1979) Motion of Charged Particles into Electric and Magnetic Fields. Nauka, Moscow.

[5] Tertychny-Dauri, V.Yu. (1996) Hyperreactive Cosmodynamics and Physics of Compact Accelerators (Nonrelativistic Model of Nuclear Electrodynamics). Institut tochnoy mechaniki i optiki, Saint-Petersburg.

[6] Tertychny-Dauri, V.Yu. (2002) Adaptive Mechanics. Kluwer Academic Publishers, Dordrecht, Boston, London.

[7] Tertychny-Dauri, V.Yu. (2004) Adaptive Stabilization in the Some Problems of Nuclear Kinetics. Izvestiya RAN. Teoriya i sistemy upravleniya, 1, 163-171.

[8] Tertychny-Dauri, V.Yu. (2006) Optimal Stabilization in the Problems of Adaptive Nuclear Kinetics. Differential Equations, 42, 3, 374-384. https://doi.org/10.1134/S0012266106030104

[9] Tertychny-Dauri, V.Yu. (2008) Hyperreactive Mechanics. In: Galamech, Vol. 3. Fizmatlit, Moscow.

[10] Tertchny-Dauri, V.Yu. (2013) Nonlinear Problems of Mechanics and Control Theory. Fizmatlit, Moscow.

[11] Tertychny-Dauri, V.Y. (2011) Hyperreactive Model in Dynamics of a Variable-Mass Point. http://arXiv.org/abs/1109.3597

[12] Tertychny-Dauri, V.Y. (2013) Principle of Completeness: Genesis of Hyperdynamics within the Theory of Reactive Motion. IOP Journal of Physics, 410, Article ID: 012077. https://doi.org/10.1088/1742-6596/410/1/012077

[13] Keepin, G.R. (1965) Physics of Nuclear Kinetics. Addison-Wesley Publishing Company, London.

[14] Krane, K.S. (1988) Introductory Nuclear Physics. John Wiley \& Sons, New York.

[15] Akhiezer, A.I. and Berestetskii, V.B. (1981) Quantum Electrodynamics. Nauka, Moscow.

[16] Berestetskii, V.B., Lifshitz, E.M. and Pitaevskii, L.P. (1968) Relativistic Quantum Theory. Nauka, Moscow.

[17] Berestetskii, V.B., Lifshitz, E.M. and Pitaevskii, L.P. (1980) Quantum Electrodynamics. Nauka, Moscow. 
[18] Mandl, F. and Shaw, G. (2010) Quantum Field Theory. John Wiley \& Sons, New York.

[19] Landau, L.D. and Lifshitz, E.M. (1976) Theoretical Physics. Vol. 5, Statistical Physics. Nauka, Moscow.

[20] Tertychny-Dauri, V.Y. (2008) Stochastic Mechanics. In: Galamech, Vol. 2, Fizmatlit, Moscow.

[21] Fermi, E. (1995) Notes on Quantum Mechanics. The University of Chicago Press, Chicago.

[22] Blokhintsev, D.I. (1983) Foundations of Quantum Mechanics. Nauka, Moscow.

\section{Notation}

* above is the transposition sign.

$\operatorname{Sp} A$ is the spur of quadratic matrix $A$ (the summarized elements of the main diagonal).

$[A, B]=A B-B A$ is the commutator of $A$ and $B$ operators with scalar product.

$\{A, B\}=A B+B A$ is the anti-commutator of $A$ and $B$ operators with scalar product.

$\{A, B\}^{\times}=A \times B-B \times A$ is the rearranged form of $A$ and $B$ operators with vector product.

All items are published in Russian except [1] [2] [3] [6] [11] [12] [13] [14] [18] [21].

Submit or recommend next manuscript to OALib Journal and we will provide best service for you:

- Publication frequency: Monthly

- 9 subject areas of science, technology and medicine

- Fair and rigorous peer-review system

- Fast publication process

- Article promotion in various social networking sites (LinkedIn, Facebook, Twitter, etc.)

- Maximum dissemination of your research work

Submit Your Paper Online: Click Here to Submit

Or Contact service@oalib.com 